\title{
Localization of Type IV Collagen $\alpha$ Chain in the Myocardium of Dilated and Hypertrophic Cardiomyopathy
}

\author{
Tomoko Watanabe, ${ }^{1} \mathrm{MD}$, Shozo Kusachi, ${ }^{1} \mathrm{MD}$, \\ Asami Yamianishi, ${ }^{1} \mathrm{MD}$, Hirofumi Kumashiro, ${ }^{,} \mathrm{MD}$, \\ Hiroshi Nunoyama, ${ }^{\prime} \mathrm{MD}$, Issei Sano, ${ }^{\prime} \mathrm{MD}$, \\ Makoto Nakahama, ${ }^{\prime} \mathrm{MD}$, Takashi Murakami, ${ }^{1} \mathrm{MD}$, \\ Ichiro NaITo, ${ }^{3} \mathrm{PhD}$, Yoshifumi Ninomrya, ${ }^{2} \mathrm{PhD}, \mathrm{MD}$, \\ and Takao TsujI,' MD
}

\section{SUMmary}

A total of $6 \alpha$ chains [ $\alpha$ l (IV) to $\alpha 6$ (IV)] have been identified in type IV collagen. We examined the localization of these chains in the myocardium of patients with dilated (DCM) and hypertrophic (HCM) cardiomyopathy. The localization of $\alpha \mathrm{l}$ (IV) $-\alpha 6$ (IV) in biopsy specimens of 5 patients with DCM and 4 with HCM was examined using immunohistochemistry with monoclonal antibodies. Both $\alpha 1$ (IV) and $\alpha 2$ (IV) immunostaining formed thin homogeneous outlines around myocytes in control hearts. In the DCM specimens, $\alpha$ l (IV) and $\alpha 2$ (IV) immunostaining formed thick and irregular patterns around myocytes. Staining for $\alpha$ l (IV) and $\alpha 2$ (IV) was also observed in some enlarged intercellular spaces. In 3 DCM hearts, moderate staining for $\alpha 1$ (IV) and $\alpha 2$ (IV) was observed in small replacement fibrotic lesions. In large replacement fibrotic lesions, no $\alpha 1$ (IV) or $\alpha 2$ (IV) staining was observed. In the HCM specimens, $\alpha 1$ (IV) and $\alpha 2$ (IV) staining formed thick homogeneous patterns around myocytes. In the enlarged intercellular spaces, no $\alpha 1$ (IV) or $\alpha 2$ (IV) staining was observed. No labeling for $\alpha 3$ (IV) $-\alpha 6$ (IV) was observed in any heart examined. In conclusion, the present results demonstrate that type IV collagen consisting of $\alpha 1$ and $\alpha 2$ chains appears in the fibrotic lesions of DCM, indicating its contribution to the development of fibrotic changes in the myocardium of DCM patients. In contrast, type IV collagen was restricted to the myocyte membrane in the HCM hearts. Fibrotic processes in the intercellular spaces may differ between DCM and HCM hearts. (Jpn Heart J 1998; 39: 753-762)

Key words: Extracellular matrix, Monoclonal antibody, Peroxidase method, Myocardial fibrosis, Left ventricular remodeling IBROTIC changes in the heart are important with respect to not only ventricular remodeling but also cardiac function. ${ }^{1-3)}$ During fibrosis, certain

From the 'First Department of Internal Medicine, 'Department of Molecular Biology and Biochemistry, Okayama University Medical School, and ${ }^{3}$ Division of Ultrastructure Research, Shigei Medical Research Institute, Okayama, Japan.

Address for correspondence: Shozo Kusachi, MD. First Department of Internal Medicine, Okayama University Medical School, 2-5-1, Shikata-cho, Okayama 700-8558, Japan.

Received for publication September 8, 1998.

Accepted October 5, 1988. 
sequential events occur, including the proliferation, migration, attachment and detachment of interstitial cells, including fibroblasts, and extracellular matrix (ECM) formation. The ECM plays a vital role in these pathologic changes. The major components of the ECM are collagens, proteoglycans, and glycoproteins.

Among the collagen subtypes, type IV collagen, a major structural protein of the basement membrane, is located in the basal membrane of myocytes. ${ }^{+1}$ Type IV collagen also possesses several binding sites that can interact with other matrix components and cellular receptors and thus contributes to basement membrane structure and cell function. ${ }^{5,6)}$ The elucidation of type IV collagen with respect to the myocardial fibrotic process is important. Using a polyclonal antibody in patients with dilated cardiomyopathy (DCM), we previously demonstrated that type IV collagen appears in fibrotic lesions where it is not related to the basement membrane. ${ }^{7)}$

Type IV collagen consists of three domains: the COL domain, which is the main part of three polypeptide $(\alpha)$ chains; the $7 \mathrm{~S}$ domain, which is located at the $\mathrm{N}$ terminal of the COL domain; and the noncollagenous (NG) domain, which is located at the $\mathrm{C}$ terminal of the chains. ${ }^{8)}$ In addition to the long-known classical $\alpha 1(\mathrm{IV})^{9)}$ and $\alpha 2$ (IV) chains, ${ }^{10)}$ recent biological studies have identified $\alpha 3$ (IV), $\alpha 4$ (IV), $\alpha 5$ (IV) and $\alpha 6$ (IV) chains. ${ }^{11-14)}$ These chains have been examined in a disease of the kidney (Goodpasture syndrome, ${ }^{11,12)}$ Alport syndrome ${ }^{13,15)}$ ) and in the normal heart. ${ }^{15}$

However, no report has examined these chains in diseased human hearts. Accordingly, we applied an immunohistochemical staining method with monoclonal antibodies to examine these chain localizations in the hearts of patients with DCM and hypertrophic cardiomyopathy (HCM).

\section{Methods}

Patients: We examined the hearts of 5 patients with DCM and 4 with HCM. The clinical characteristics of the patients studied are summarized in Table I. DCM and HCM were diagnosed according to the diagnostic criteria of the World Health Organization/International Society and Federation of Cardiology (WHO/ISFC). ${ }^{16)}$ Endomyocardial biopsies were performed in patients with DCM and HCM after obtaining their informed consent. Samples were obtained from the postero-lateral wall of the left ventricle. Three to five biopsy specimens were obtained from each patient; two or three specimens were stained with hematoxylin-eosin and Masson-Trichrome for light microscope examination, and the remaining specimens were subjected to immunohistochemistry. Left ventricular specimens obtained from two autopsy cases without any overt heart disease served as controls. 
Table I. Characteristics of the Study Pateints

\begin{tabular}{|c|c|c|c|c|c|c|}
\hline & \multirow{2}{*}{ Age } & \multirow{2}{*}{ Gender } & \multicolumn{3}{|c|}{ Echocardiography } & \multirow{2}{*}{$\frac{\text { Chest X-ray }}{\text { CTR }}$} \\
\hline & & & LVDd & LVEF & LVPUth & \\
\hline \multicolumn{7}{|l|}{ DCM } \\
\hline \#1 & 58 & $\mathrm{~m}$ & 65 & 20 & 9 & 48 \\
\hline$\# 2$ & 24 & $\mathrm{~m}$ & 83 & 13 & 8 & 70 \\
\hline$\# 3$ & 74 & $\mathrm{~m}$ & 70 & 35 & 7 & 54 \\
\hline$\# 4$ & 41 & $\mathrm{~m}$ & 67 & 31 & 11 & 64 \\
\hline$\# 5$ & 57 & $\mathrm{f}$ & 49 & 33 & 9 & 60 \\
\hline \multicolumn{7}{|l|}{$\mathrm{HCM}$} \\
\hline \#1 & 47 & $\mathrm{~m}$ & 52 & 73 & 19 & 57 \\
\hline$\# 2$ & 48 & $\mathrm{~m}$ & 50 & 65 & 18 & 51 \\
\hline \#3 & 43 & $\mathrm{~m}$ & 49 & 73 & 14 & 49 \\
\hline$\# 4$ & 35 & $f$ & 32 & 88 & 23 & 59 \\
\hline
\end{tabular}

DCM = dilated cardiomyopathy; HCM = hypertrophic cardiomyopathy; LVDd = left ventricular end-diastolic dimension; $L V E F=$ left ventricular ejection fraction; $L V P W$ th $=$ left ventricular posterior wall thickness; CTR = cardiothoracic ratio.

Table II. The $\alpha$ Chain-specific Monoclonal Antibodies Lsed in This Study

\begin{tabular}{lcll}
\hline Name & $\alpha$ chain specificity & Isotype & Epitope \\
\hline H11 & $\alpha$ l & IgG $2 a$ & KKPTPSTL \\
H22 & $\alpha 2$ & IgG $2 a, \mathbf{k}$ & DTLKAGLIR \\
H31 & $\alpha 3$ & IgG $2 a, \mathbf{k}$ & IPSTVKA \\
H43 & $\alpha 4$ & IgG $2 b, \mathbf{k}$ & PAPDTLKE \\
H52 & $\alpha 5$ & IgG $2 b, \mathbf{k}$ & SKPQSETL \\
H63 & $\alpha 6$ & IgG $2 a, \mathbf{k}$ & GELP \\
\hline
\end{tabular}

Conventional light microscopic examination: The cardiac biopsy tissue was fixed with $10 \%$ buffered formalin and embedded in paraffin. Each specimen was then cut into $6-\mu \mathrm{m}$ sections that were stained with hematoxylin-eosin and Masson-Trichrome.

Immunohistochemistry: Rat anti-human monoclonal antibodies (MoABs $\mathrm{H} 11$ to $\mathrm{H} 64)$ which recognize the $\alpha$ l to $\alpha 6$ chains of type IV collagen [ $\alpha 1$ (IV) to $\alpha 6$ (IV)] were used. These antibodies were raised against a synthetic peptide of a nonconsensus amino acid sequence close to the carboxyl terminal of the human $\alpha$ (IV) NC domain using rat medial iliac lymph nodes. ${ }^{17)}$ Specificity was confirmed by an enzyme-linked immunosorbent assay and an epitope analysis using multipeptide screening. ${ }^{17.18)}$ The $\alpha(\mathrm{IV})$ chain specificity, isotype and epitope of the MoABs are listed in Table II.

For immunohistochemistry, we employed the immunoperoxidase method. The sections were deparaffinized in xylene and dehydrated through graded ethanol solutions. The sections were then washed for 5 minutes $(\times 2)$ in deionized water. Because paraffin-embedded sections were used, antigen retrieval was done before immunostaining. ${ }^{19}$ ) Deparaffinized sections were set in a staining chamber filled with $0.2 \mathrm{M} \mathrm{HCl}$ (the $\mathrm{pH}$ was approximately 1.0). The chamber was set in 
a small-sized autoclave (KT-2322, ALP Co., Tokyo, Japan), and the antigen retrieval was carried out by heating the sections to 110 to $127^{\circ} \mathrm{C}$ for 6 minutes (the suitable temperature was varied for the different MoABs). The sections were then washed 5 minutes $(\times 2)$ in deionized water. Thereafter, endogenous peroxidase activity was blocked by $3 \%$ hydrogen peroxide for 5 minutes, and the sections were washed with Tris-buffered saline (TBS) for 5 minutes.

After antigen retrieval, the sections were incubated with the MoABs listed in Table II, for 30-60 minutes at room temperature. The sections were treated with the secondary antibody (a mixture of anti-mouse IgG serum and anti-rabbit IgG serum) for 10 minutes at room temperature, with the use of an LSAB2 kit (DAKO, Carpinteria, CA, USA). ${ }^{20)}$ The sections were washed in TBS for 10 minutes and incubated with strept-avidin peroxidase for 10 minutes at room temperature. They were then washed in TBS for $10 \mathrm{~min}$, and colored with $3 \% 3$ amino-9-ethylcarbazole in $\mathrm{N}$-dimethylformamide.

Negative control staining with TBS instead of primary antibody and an immunoabsorption test using the corresponding peptide were performed.

Analysis: We analyzed the localization of each $\alpha$ chain of type IV collagen in intercellular spaces and replacement fibrotic lesions. The staining results were semi-quantified and graded as follows: - , none; + , slight but apparent; ++ , moderate (staining grade between + and ++ ); ++ , strong. All assessments were done by 2 investigators in a single-blind manner. When the two observers disagreed, an additional observer checked the samples.

\section{Results}

Conventional light microscopic examination: The light microscopic findings of the 9 patients' heart biopsy samples are summarized in Table III. Consider-

Table III. Light Microscopic Findings of the Endomyocardial Biopsy Specimens from DC.M and HC.M Patients

\begin{tabular}{|c|c|c|c|c|c|}
\hline & $\begin{array}{c}\text { Myonyte } \\
\text { degeneration }\end{array}$ & $\begin{array}{c}\text { Myocyte } \\
\text { hypertrophy }\end{array}$ & $\begin{array}{l}\text { Enlargement of } \\
\text { endomysium }\end{array}$ & $\begin{array}{l}\text { Replacement } \\
\text { fibrosis }\end{array}$ & $\begin{array}{l}\text { Myocyte } \\
\text { disarray }\end{array}$ \\
\hline \multicolumn{6}{|l|}{ DCM } \\
\hline$\# 1$ & + & + & H & + & + \\
\hline \#2 & $H$ & + & $H$ & + & H \\
\hline$\# 3$ & H+ & +t & + & + & + \\
\hline$\# 4$ & + & + & + & + & + \\
\hline$\# 5$ & + & + & + & + & H \\
\hline \multicolumn{6}{|l|}{$\mathrm{HCM}$} \\
\hline$\# 1$ & + & $H$ & + & - & + \\
\hline$\# 2$ & + & H & + & - & + \\
\hline \#3 & + & + & + & + & $H$ \\
\hline$\# 4$ & + & $H$ & + & - & t+t \\
\hline
\end{tabular}


Table IV. Immunohistochemical Staining Results for $\alpha 1$ and $\alpha 2$ Chains of Type IV Collagen

\begin{tabular}{|c|c|c|c|c|c|c|c|c|}
\hline \multirow[t]{2}{*}{ DCM } & \multirow{2}{*}{$\begin{array}{l}\text { Aroud } \\
\text { myocytes }\end{array}$} & \multicolumn{2}{|c|}{$\begin{array}{l}\text { Intercellular } \\
\text { spaces }\end{array}$} & \multicolumn{2}{|c|}{$\begin{array}{l}\text { Replacement } \\
\text { fibrotic lesions }\end{array}$} & \multirow[t]{2}{*}{$\mathrm{HCM}$} & \multirow{2}{*}{$\begin{array}{l}\text { Around } \\
\text { myocytes }\end{array}$} & \multirow{2}{*}{$\begin{array}{c}\text { Intercellular } \\
\text { spaces }\end{array}$} \\
\hline & & Dilated & Non-dilated & Small & Large & & & \\
\hline$\# 1$ & thick and irregular & + & + & + & 1 & $\# 1$ & thick & - \\
\hline \#2 & thick and irregular & + & + & + & - & $\# 2$ & thick & - \\
\hline \#3 & thick and irregular & +4 & + & + & - & $\# 3$ & thick & - \\
\hline \#4 & thick and irregular & + & + & - & - & $\# 4$ & thick & - \\
\hline \#5 & thick and irregular & + & + & - & - & & & \\
\hline
\end{tabular}

able fibrosis associated with myocyte necrosis consistent with the characteristics of DCM were observed in all DCM cases. The intercellular spaces in all 5 cases were wider than those in control specimens. In the 4 patients with HCM, myocyte hypertrophy and various degrees of myocyte disarray were observed.

Immunohistochemical staining: The negative control staining with TBS and the antibody absorption tests confirmed the specificity of the staining results. Table IV summarizes the results of the immunohistochemical staining.

In the control hearts, positive thin-layer staining for $\alpha \mathrm{l}$ (IV) and $\alpha 2$ (IV) was observed around myocytes. The staining pattern was homogeneous (Figure $1 \mathrm{~A}$ and $1 \mathrm{~B})$.

DCM: Identical staining pattens for $\alpha$ l (IV) and $\alpha 2$ (IV) were observed. The $\alpha 1$ (IV) and $\alpha 2$ (IV) staining around myocytes was thick and irregular (Figure 2) compared to that in the control hearts (Figure l). $\alpha$ l (IV) and $\alpha 2$ (IV) staining was detected in the small replacement fibrotic lesions but not in the large replacement fibrotic lesions. In some enlarged intercellular spaces near the replacement fibrotic lesions, $\alpha \mathrm{l}$ (IV) and $\alpha 2$ (IV) staining was observed (Figure 2B-2D). The staining pattern varied from lesion to lesion. $\alpha 3$ (IV) $-\alpha 6$ (IV) labeling was not observed in any lesion in any of the 5 DCM hearts examined.

HCM: The $\alpha$ l (IV) and $\alpha 2$ (IV) staining formed a thick band around myocytes (Figure 3B-3C). In the intercellular spaces, $\alpha$ l (IV) and $\alpha 2$ (IV) labeling was not observed. $\alpha 3$ (IV) $\alpha 6$ (IV) staining was not observed in any lesion in the $4 \mathrm{HCM}$ hearts examined.

\section{Discussion}

The present study demonstrated that type IV collagen composed of $\alpha 1$ and $\alpha 2$ chains appears in the fibrotic lesion of DCM hearts, where it is not restricted to the basement membrane, indicating that it contributes to fibrotic processes in DCM hearts. In the HCM heart, positive staining for type IV collagen $\alpha$ chain was not observed in the intercellular fibrotic spaces, indicating that the ECM formation processes in enlarged intercellular spaces of HCM hearts may be dif- 


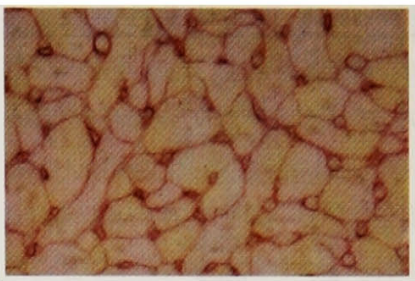

A

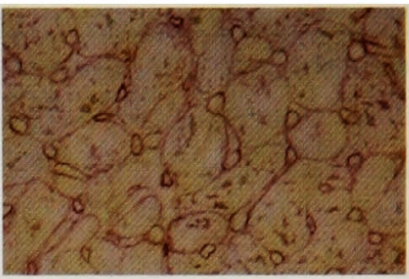

B

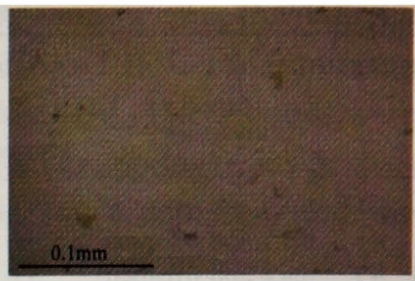

C

Figure 1. A: Immunostaining for $\alpha$, IV in a control heart. A thin-layer of homogeneous staining is observed around myocytes. B: Immunostaining for $\alpha 2$, IV / in a control heart. A staining pattern similar to that of $\alpha \mathrm{l} \mid \mathrm{IV}$ was seen. C: Negative control staining in a control heart.

$\mathbf{A}$
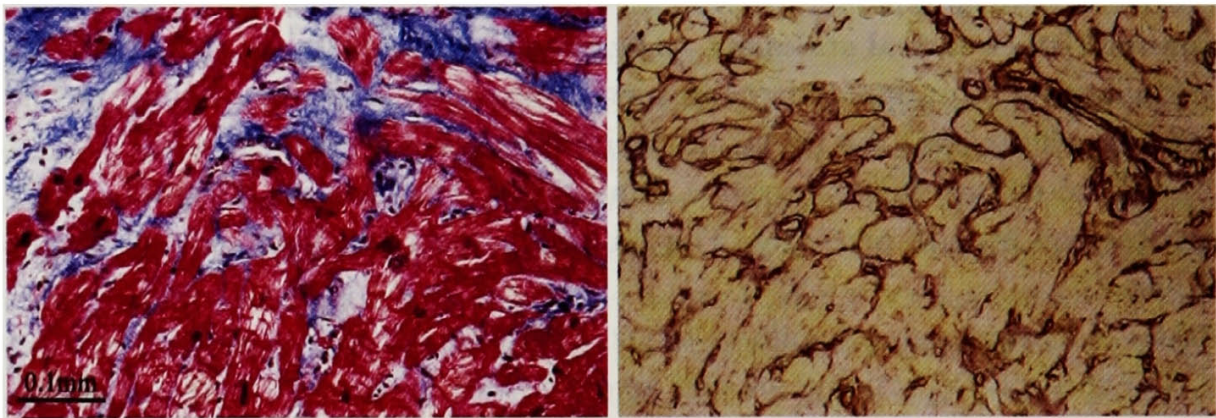

B
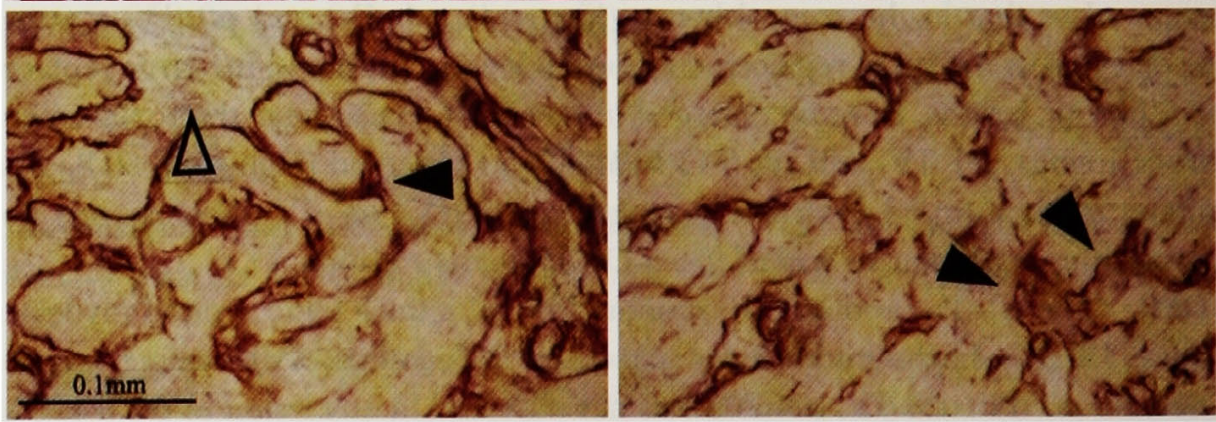

D

Figure 2. I ( i M heart I $)(: M$ Patient \#1). A: Masson-Trichrome staining. B-D: Immunostaining for $\alpha_{2}{ }_{2}[V\rangle$. A: Considerable fibrotic changes with myocyte degeneration can be seen. B: Low-power field of the immunostaining. C: High-power field. Open triangle indicates a large replacement fibrotic lesion where no positive staining can be seen. Closed triangle indicates an enlarged intercellular space near the replacement fibrotic lesion where positive staining is seen. D: High-power field. Closed triangles indicate small replacement fibrotic lesions where moderately positive staining can be seen.

ferent from those in the fibrotic lesions of DCM hearts.

Both $\alpha$ l (IV) and $\alpha 2$ (IV) surrounded myocytes in the control hearts (Figure 
$\mathbf{A}$
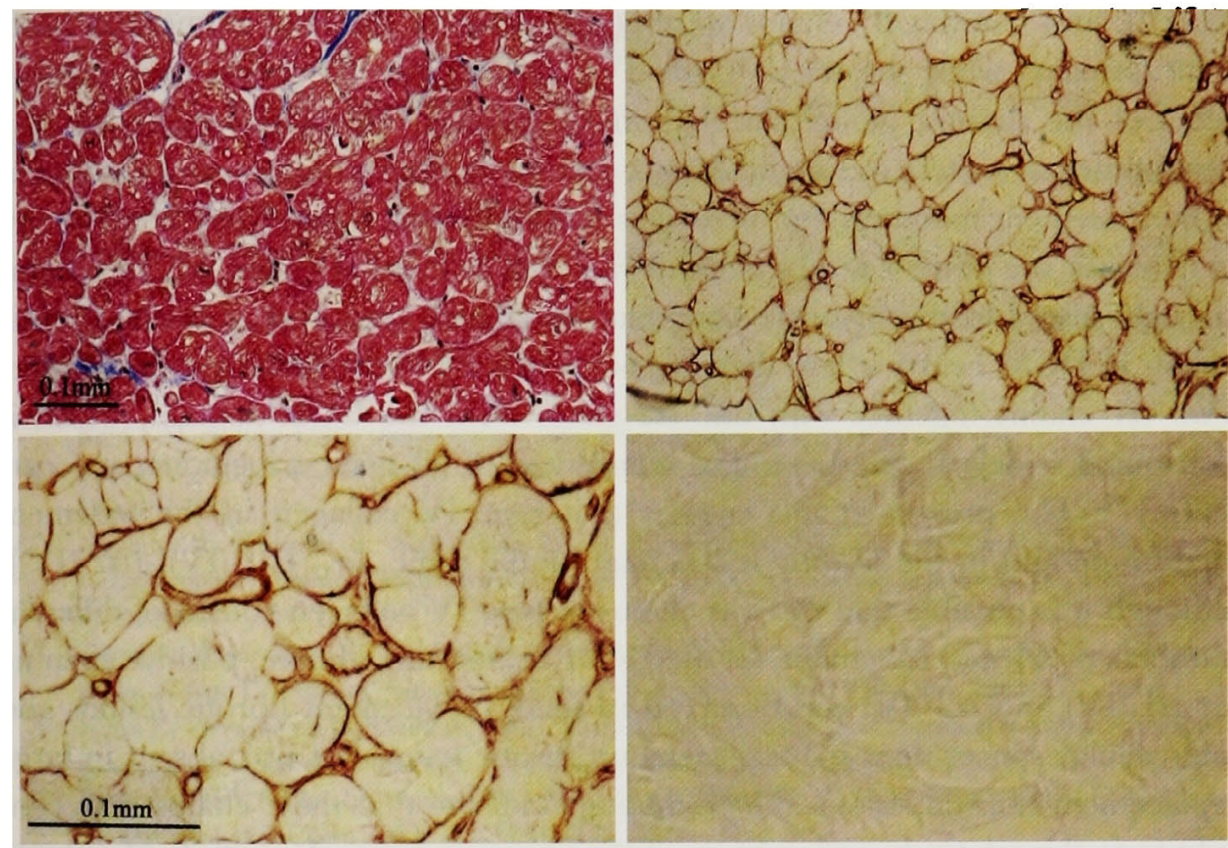

Figure 3. HCM heart (HCiN Patient \#1). A: Masson-Trichrome staining. B and $\mathbf{C}$ : Immunostaining for $\alpha 2 ; \mathrm{IV}$. Enlargement of intracellular spaces can be seen in $\mathbf{B}$ (low-power field) and $\mathbf{C}$ (high-power field). Thick and homogeneous staining can be seen around the myocytes. No staining is observed in intercellular spaces. D: Negative control staining. No staining is observed.

1A, 1B). Ninomiya et al. ${ }^{16)}$ using indirect immunofluorescence staining with monoclonal antibodies, showed that both $\alpha$ l (IV) and $\alpha 2$ (IV) staining appeared in the basement membrane of myocytes, but $\alpha 3$ (IV), $\alpha 4$ (IV), $\alpha 5$ (IV) or $\alpha 6$ (IV) labeling was not present in normal human hearts at autopsy. These observations are consistent with the present results observed in control hearts, indicating that the method employed was suitable for this study.

It is not known how many different isoforms of type IV collagen exist and how these chains contribute to the COL domain composition for different isoforms of type IV collagen. In the pathologic course of myocardial fibrosis, cell migration, matrix deposition, and tissue remodeling are essential processes, and resemble those of embryonic development. Brown et al. ${ }^{21)}$ used in situ hybridization to examine the role of fibronectin in the cutaneous wound healing process, and observed embryonic fibronectin gene expression. Laminin, another major component of the basement membrane, has several isoforms consisting of different chains. ${ }^{22)}$ Previous studies demonstrated that a developmental switch occurs in laminin $\beta$ chain..$^{23-25)}$ Mincr et al. ${ }^{26)}$ examined typc IV collagen and laminin chains in rodent kidney using immunohistochemistry and found that the $\alpha$ l IV), 
$\alpha 2$ (IV), and laminin $\beta 1$ chains were components of the fetal glomerular basement laminae, while the $\alpha 4$ (IV), $\alpha 5$ (IV), and laminin $\beta 2$ chains were components of the mature glomerular basement laminae. Given this evidence of a developmental switch, one could expect that the $\alpha$ chains of the type IV collagen in the basement membrane of myocytes and in fibrotic lesions may be different. In contrast, the present results demonstrate that both $\alpha$ l (IV) and $\alpha 2$ (IV) chains are colocalized to the myocyte basement membrane and appear in the fibrotic lesions in DCM hearts where they are not restricted to the basement membrane. Two $\alpha$ l chains together with one $\alpha 2$ chain form the COL domain, which is distributed widely in the basement membrane of all tissues, including myocardium. ${ }^{27,28)}$ The present results suggest that type IV collagen in the basement membrane and in the fibrotic lesion may be the same, i.e., $\left[\alpha 1(\text { IV) }]_{2}[\alpha 2\right.$ (IV)], although it is possible that three $\alpha$ chains of type IV collagen consist of different numbers of $\alpha 1$ and $\alpha 2$ chains. In the DCM hearts studied here, positive staining for $\alpha 1$ (IV) and $\alpha 2$ (IV) was observed in small replacement fibrotic lesions and intercellular spaces near the replacement fibrotic lesion but was absent in large replacement fibrotic lesions. These findings indicate that these chains may contribute to the relatively early phases of the fibrotic process. In a study of autopsy cases involving myocardial infarction, ${ }^{29}$ ) type IV collagen was distributed in granulation tissue in the early phase of the infarct and was not restricted to the basement membrane, a finding similar to our observations in DCM hearts.

In the HCM studied here, positive staining for type IV collagen $\alpha$ chain was not observed in intercellular spaces, indicating that type IV collagen does not contribute to the intercellular fibrotic process in HCM. The ECM formation processes in enlarged intercellular spaces of HCM hearts may be different from those in the fibrotic lesions of DCM.

We were unable to identify the infiltrating cells with the present immunohistochemical technique. In the biopsy specimens from DCM hearts, hematoxylin-eosin staining revealed that several fibroblasts were present in the enlarged intercellular spaces and replacement fibrotic lesions. Type IV collagen is produced by several cell lines, including myocytes and fibroblasts. ${ }^{30}$ Fibroblasts may be one of the type of cells that produce both $\alpha$ l (IV) and $\alpha 2$ (IV) in fibrotic lesions. The thick staining for $\alpha \mathrm{l}$ (IV) and $\alpha 2$ (IV) observed around myocytes in the present HCM hearts suggests that the myocytes might have produced these chains.

In conclusion, we have demonstrated that among the $6 \alpha$ chains of type IV collagen, the $\alpha 1$ and $\alpha 2$ chains appear in the fibrotic lesions of DCM where they were not restricted to the basement membrane. In contrast, type IV collagen was restricted to the myocyte membrane in HCM hearts. 


\section{References}

1. Weber KT. Cardiac interstitium in health and disease: the fibrillar collagen network. J Am Coll Cardiol 1989; 13: 1637-52.

2. Weisman HF, Healy B. Myocardial infarct expansion, infarct extension, and reinfarction: pathophysiologic concepts. Prog Cardiovasc Dis 1987; 30: 73-110.

3. Pfeffer NLA. Braunwald E. Ventricular remodeling after myocardial infarction. Circulation 1990; 81: 1161-72.

4. Speiser B, Riess FC, Schaper J. The extracellular matrix in human myocardium. Part I: Collagen I, III, IV, and VI. Cardioscience 1991; 2: 225-32.

5. Furthmayr H. Basement membrane collagen: structure, assembly, and biosynthesis. In: Zern MA, Reid KM, editors: Extracellular matrix, New York: Marcel Dekker, 1993: 149-85.

6. Timpl R. Structure and biological activity of basement membrane components. Eur J Biochem 1989; 180: $487-502$.

7. Nogami K, Kusachi S, Nunoyama $\mathbf{H}$, et al. Extracellular matrix components in dilated cardiomyopathy: immunohistochemical study of endomyocardial biopsy specimens. Jpn Heart J 1996; 37: 483-94.

8. Timpl R, Wiedemann H, Van Delden V. Furthmayr H, Kühhn K. A network model for the organization of type IV collagen molecules in basement membranes. Eur J Biochem 1981; 120: 203-11.

9. Kühn $\mathrm{K}$. Wiedemann $\mathrm{H}$, Timpl R, et al. Macromolecular structure of basement membrane collagens: identification of $7 \mathrm{~s}$ collagen as a cross-linking domain of type IV collagen. FEBS Let 1981; 125: 123-8.

10. Bächinger HP, Fessler LI, Fessler LH. Mouse procollagen IV. Characterization and supramolecular association. J Biol Chem 1982; 257: 9796-803.

11. Butkowski R, Wieslander J, Wilson BJ, Barr JF, Noelken ME, Hudson BG. Properties of the globular domain of type IV collagen and its relationship to the Goodpasture antigen. J Biol Chem 1985; 260: $3739-45$.

12. Butkowski RJ, Langeveld JPM, Wieslander J, Hamilton J, Hudson BG. Localization of the Goodpasture epitope to a novel chain of basement membrane collagen. J Biol Chem 1987; 262: 78747.

13. Hostikka SL, Eddy RL, Byers MG, Höyhtya M, Shows TB, Tryggvason $\mathrm{K}$. Identification of a distinct type IV collagen $\alpha$ chain with restricted kidney distribution and assignment of its gene to the locus of $\mathrm{X}$ chromosome-linked Alport syndrome. Proc Natl Acad Sci USA 1990; 87: 1606-10.

14. Zhou J, Mochizuki T, Smeets $H$, et al. Deletion of the paired $\alpha 5$ (IV) and $\alpha 3$ (IV) collagen genes in inherited smooth muscle tumors. Science 1993; 261: 1167-9.

15. Ninomiya $\mathrm{Y}$, Kagawa $\mathrm{M}$, et al. Differential expression of two distinct membrane collagen genes, COL4A6 and COL4A4, demonstrated by immunofluorescence staining using peptide-specific monoclonal antibodies. J Cell Biol 1995; 130: 1219-29.

16. Richardson P, McKenna W, Bristow MI, et al. Report of the 1995 World Health Organization/ International Society and Federation of Cardiology Task Force on the Definition and Classification of Cardiomyopathies. Circulation 1996; 93: 841-2.

17. Sado $Y$, Kagawa M, Kishiro $Y$, et al. Establishment by the rat lymph node method of epitope-defined monoclonal antibodies recognizing the six different $\alpha$ chains of human type IV collagen. Histochem Cell Biol 1995; 104: 267-75.

19. Sado Y, Kagawa M, Rauf S, Naito I, Moritoh C, Okigaki T. Isologous monoclonal antibodies can induce anti-GMB glomerulonephritis in rats. J Pathol 1993; 168: 221-7.

20. Naito I, Kawai S, Nomura S, Sado Y, Osawa G, Japanese Alport Network. Relationship between COL4A5 gene mutation and distribution of type IV collagen in male X-linked Alport syndrome. Kidney Int 1990; 50: 304-11.

21. Brown LF, Dubin D, Lavigne L, Logan B, Dcorak HF, Van De Water L. Macrophages and fibroblasts express embryonic fibronectins during cutaneous wound healing. Am J Pathol 1993; 142: 793-801.

22. Burgeson R, Chiquet M, Deutmann R, Ekblom P, Engel J, Kleinman H, et al. A new nomenclature for the laminins. Matrix Biol 1994; 14: 209-11.

23. Bernier SM, Utani A, Sugiyama S, Doi T, Polistina C, Yamada Y. Cloning and expression of laminin 
$\alpha 2$ chain (M-Chain) in the mouse. Matrix Biology 1994; 14: 447-55.

24. Nissinen M, Vuolteenaha R, Boot-Handford R, Kallunk P, Tryggvason K. Primary structure of the human laminin A chain. Biochem J 1991; 276: 369-79.

25. Katayama $\mathbf{S}$, Abe $\mathbf{M}$, Inaba $\mathbf{M}$, Itabashi A, Ishii J. Effects of captril or nitrendipine on left ventricular collagen or laminin $\beta 2$ gene expression. Jpn Heart J 1993; 34: 773-83.

26. Miner JH, Sanes JR. Collagen IV $\alpha 3, \alpha 4$ and $\alpha 5$ chains in rodent basal laminae: sequence, distribution, association with laminins, and developmental switches. J Cell Biol 1994; 127: 879-91.

27. Trueb B, Schreier T, Bruckner P, Winterhalter K. Type VI collagen represents a major fraction of connective tissue collagens. Eur J Biochem 1987; 166: 699-703.

28. Mayne R, Wiedemann H, DessauW, Von den Mark K, Bruckner P. Structural and immunological characterization of type IV collagen isolated from chicken tissues. Eur J Biochem 1982; 126: 417-23.

29. Shekhonin BV, Domogatsky SP, Idelson GL, Koteliansky VE. Participance of fibronectin and various collagen types in the formation of fibrous extracellular matrix in cardiosclerosis. J Mol Cell Cardiol 1988; 20: 501-8.

30. Eghbali M, Weber KT. Collagen and the myocardium: fibrillar structure, biosynthesis and degradation in relation to hypertrophy and its regression. Mol Cell Biochem 1990; 96: 1-14. 